\title{
Enzyme-Histochemical Observation on the Kidney of Stroke-prone SHR
}

\author{
Akio Shino, Akinobu Nagaoka, and Hisashi Imatsuka
}

It is well known that SHRSP develops severe hypertension accompanying reno- and cerebrovascular lesions. Renal vascular lesions appear to accelerate the onset of stroke through either exaggerating hypertension or disturbing water and mineral metabolism. In the present study, enzyme histochemistry of the kidney was performed to examine morphological and/or functional alterations of tubules prior to the onset of stroke.

Materials and Methods:

Ten to 17 weeks old male SHRSP and Wistar Kyoto (WK) rats were used. Their blood pressures were determined by tail-pulse pickup method on the day of sacrifice. In the case of rats which developed stroke signs during the experimental periods, they were autopsied within 24 hours to confirm the presence of cerebral lesions. The kidneys were removed from rats under anesthesia and then sliced at 3-5 $\mathrm{mm}$ in thickness. Some of slices were freezed with dryice-acetone, others were fixed in $0.1 \mathrm{M}$ cacodylate-buffered $4 \%$ paraformaldehyde. Enzyme histochemistry was performed to detect acid phosphatase (ACPase), alkaline phosphatase (ALPase), succinic dehydrogenase (SDH), paranitrophenyl phosphatase (PNPPase), and lactate dehydrogenase (LDH). Some of the tissues fixed in $4 \%$ paraformaldehyde were embedded in paraffin and stained with H.E., PAS and phosphotungstic acid hematoxylin (PTAH). Electron microscopical observations of the kidneys were also performed with some rats.

\section{Results:}

There was no alteration of enzyme activities in the kidney from 10 weeks old SHRSP when compared with those of WK rats. In 13 weeks old SHRSP, ALP ase activity of brush border and SDH activity of tubular cells were slightly decreased in proximal tubules. LDH activity was also decreased in smooth muscle cells of some degenerated arteries.

The above enzymatic changes were more advanced in 14-17 weeks old SHRSP. Furthermore, decreases in tubular enzyme activities were distributed from proximal to distal parts. Concomitantly, increased ACPase activity was evidenced with tubular cells. These alterations suggested degenerative changes of tubules. PNPPase activity of tubules was markedly decreased. These changes appeared to be more prominent in rats with stroke.

Histology of the kidney: In SHRSP aged 10-13 weeks, there was occasionally observed focal necrosis of interlobular arteries. Fibrinoid necrosis and proliferative arteriosclerosis accompanying glomerular alteration and tubular degeneration were observed in the kidney from 14-17 weeks old SHRSP. By the electron micro-

From the Biological Research Laboratories, Central Research Division, Takeda Chemical Industries, Osaka. 
scopy, destruction of brush borders, swelling of metochondria and decreased density of cytoplasmic matrix were observed in proximal tubules of these rats.

\section{Conclusions :}

Our histochemical observations revealed decreases in several enzyme activities of the kidneys of SHRSP prior to the onset of stroke. At first, degeneration of brush borders and mitochondria in the proximal tubular cells were observed in 13 weeks old SHRSP. With the advance of age, PNPPase and SDH activities in distal tubules and thick ascending limb of Henle were markedly decreased. The decreased activity of PNPPase suggests impaired functions in ion transport system of these tubules, since this enzyme is comparable to that of $\mathrm{Na}^{+}-\mathrm{K}^{+}-\mathrm{ATPase}$. Therefore, elevation of PRA or natriurea of SHR evidenced by Willis et al (J Lab Glin Med 87:265, 1976) seem to produce partly by impaired $\mathrm{Na}^{+}$reabsorption. Furthermore, thickening and necrotic changes of arterial walls were occasionally observed in the kidney from 10 weeks old SHRSP. In addition, ultrastructural alterations observed in the proximal tubules were very similar to those observed in tubules of kidney exposed to experimental ischemia. Thus, the tubular changes observed in SHRSP appear to be secondary to ischemia due to arterial changes caused by severe hypertension. 